Running Head: Event Markers and Feeling of Time

\title{
1995 Feels so Close Yet so Far:
}

\section{The Effect of “Event Markers” on Subjective Feelings of Elapsed Time}

\author{
Gal Zauberman ${ }^{1}$, Jonathan Levav${ }^{2}$, Kristin Diehl ${ }^{3}$, Rajesh Bhargave ${ }^{1}$ \\ ${ }^{1}$ University of Pennsylvania $\quad{ }^{2}$ Columbia University ${ }^{3}$ University of Southern California
}

WORD COUNT: 3962

NUMBER OF REFERENCES: 14

Please address correspondence to:

Gal Zauberman

700 Jon M. Huntsman Hall

3730 Walnut Street

University of Pennsylvania

Philadelphia , PA 19104

Phone: (215) 573-0558

Fax: (215) 898-2534

zauberman@wharton.upenn.edu 


\begin{abstract}
Why do some events feel more distant than others? Prior research suggests that characteristics of the event itself can affect the estimated date of its occurrence. Our work differs in that we focus on how characteristics of the time interval following the target event can affect people's feelings of elapsed time, as revealed in their everyday expressions of how recent or distant an event seems. We argue that a time interval that is punctuated by a greater number of events precipitated by the target event (“event markers”) will make the target event feel more distant. In three studies we find that the greater the number of event markers, the more distant an event feels. The effect of markers is independent of other characteristics of the event, such as its memorability, its emotionality, and its estimated date, suggesting that this effect is distinct from established biases in date and duration estimation.
\end{abstract}

[150 words] 


\title{
1995 Feels so Close Yet so Far:
}

\section{The Effect of “Event Markers” on Subjective Feelings of Elapsed Time}

\author{
“Time ... persists merely as a consequence of the events taking place in it." \\ Martin Heidegger (1992/1924), The Concept of Time, p.3E.
}

In common parlance people often express elapsed time in terms of a subjective sensation or "feeling" rather than a precise duration or date estimate. For instance, when returning to a restaurant a person might remark, “it feels like we were last here ages ago.” People express such feelings even for very meaningful events whose actual date of occurrence is known. For example, on a recent trip to the memorial of former Israeli prime-minister Yitzhak Rabin, who was felled by an assassin's bullet in 1995, the wife of the first author observed that Rabin's assassination felt like it had occurred very recently, whereas the 1995 birth of her twins felt very distant. In this paper we ask: What makes some events feel more distant than others?

Prior research suggests that judgments of event dates and duration are driven by characteristics of the event itself (e.g., its vividness). In contrast, we show that subjective feelings of elapsed time, or a person's sense of temporal distance from a past event, are not only affected by characteristics of the event itself, but also by characteristics of the interval following the target event.

There are two categories of information that people can rely on when assessing elapsed time since a target event. The first category is information that can be retrieved about the event itself; the second category is information that can be retrieved about the time interval since the 
event. Prior research on time perception has focused on the first category. For example, strength-of-memory models demonstrate that accessible (Brown, Rips, \& Shevell, 1985) and emotional events (Bratfisch, Ekman, Lundberg, \& Kruger, 1971) create stronger traces in memory, which lead the dates of more distant events to be estimated as more recent, a phenomenon labeled “forward telescoping” (for reviews see Friedman, 1993; Morwitz, 1997). Other work suggests that contextual cues about the event can be used to reconstruct its placement in time (e.g., Friedman, 1996). Remembering that one was wearing a heavy coat, for example, indicates that the event occurred in the winter months. Beliefs about causality can also be used to judge elapsed time; people judge causally related events to be more temporally proximal (Faro, Leclerc, \& Hastie 2005).

In contrast to these approaches, our work focuses on how characteristics of the time interval following the target event can affect people's sense of elapsed time since that event. We focus on events precipitated by the target event, which we call "event markers." For instance, for the target event "birth of a child," event markers include visits to the doctor, diaper changes, play-dates in the park, piano recitals, and many other events that a parent might bring to mind. In contrast, the assassination of a Prime Minister may bring to mind fewer event markers, in part because the political changes triggered by this target event may not be as accessible. We argue that a time interval punctuated by a greater number of event markers will make its precipitating event feel more distant. As such, our prediction departs from strength-of-memory models: whereas these models predict that recalling more aspects related to a target event results in people judging that event to be more recent, we posit that accessing more events that occurred subsequent to the target event results in that event feeling more distant. 
Our prediction is related, in part, to research indicating that the perceived duration of an event depends on its content and composition. For instance, short episodes are judged to be longer in retrospect when they are more complex (see Block \& Zakay, 1997), when they are less routine (Avni-Babad \& Ritov, 2003), or when they provide greater contextual change (Zakay \& Block, 2004). Similarly, episodes that are "filled” with a greater number of components are perceived to be longer, a phenomenon known as the filled-duration illusion (Ornstein, 1969). For instance, an interval is judged to be longer if it is interrupted by 60 words compared to 30 words (Block, 1974). The number of words provides a cue to infer the duration of the target interval. Although event markers exert a similar influence on people’s feelings of elapsed time, they are distinct in two important ways. First, markers do not occur in the course of the target event, as in previous research, but rather following that event. Second, when people judge feelings of elapsed time in our studies, the interval between the target event and their judgment can last several months (rather than several minutes, as in previous research), such that event markers are likely to occupy a far smaller proportion of the overall interval.

In summary, we predict that even events that 1) occurred approximately at the same time and 2) are similar in terms of characteristics of the event itself (e.g., vividness), may still differ in how distant they feel and that this difference can be explained by our concept of event markers. We suggest that, over and above a target event's characteristics, the greater the number of markers associated with an event, the greater the feeling of elapsed time since that event. Moreover, we argue that the effect of markers on subjective feelings of elapsed time holds even after controlling for the estimated date of the event, suggesting that our effect is psychologically distinct from previously established biases in date and duration estimation. 
We test our hypothesis in three studies that include both public and autobiographical events that occurred weeks, months, and years in the past. We rely on correlational survey data measuring event markers for public events (study 1) and manipulate both the number of markers that follow a purposely created target event (study 3) as well as the accessibility of markers following an autobiographical event (study 2). An important feature of our studies is that we control for memorability, emotionality and estimated dates, thus establishing the effect of event markers over and above such event-specific characteristics.

\section{Study 1: Public Events}

The goal of this study was to test our event marker hypothesis using a series of wellknown public events. We measured the number of subsequent events (markers) that respondents associated with eight public events in domains spanning political and cultural events nationally and internationally (see Appendix for a list of events and dates). These target events took place between November, 2004 and September, 2005, 16 to 26 months prior to the administration of this study. We hypothesize that events associated with a greater number of markers will feel more distant than events associated with fewer markers.

\section{Methods}

One hundred and eighty-five University of Pennsylvania students (104 female, 81 male) participated in this study as part of a larger session for which they were paid. The survey consisted of five blocks of judgments. Participants first indicated their subjective feeling of elapsed time for all eight target events, and then rated the extent to which each target event triggered subsequent events, the extent to which each target event was memorable and emotional 
to them and, finally, the date in which they believed each target event had actually occurred. Order of events in the survey was counterbalanced.

Participants’ subjective feeling of elapsed time was measured on a 15-point scale anchored by "feels very recent” (1) and "feels very distant” (15). The extent to which participants perceived that the target event triggered subsequent events was also measured on a 15-point scale ranging from “feels like it triggered few events" (1) to "feels like it triggered many events” (15). As in prior research (e.g., Burt \& Kemp, 1991), memory of the event was measured on a 1 (“not at all”) to 7 (“perfectly”) scale. Emotionality of the event was also measured on a 7-point scale, ranging from 1 (“not at all emotional”) to 7 (“extremely emotional”). Finally, participants indicated the month and year in which they believed that the target event had occurred; this was used to calculate the estimated number of months passed. Where participants only provided a year (e.g., 2004) we calculated the number of months elapsed since June of that year. Four participants did not provide a date for any of the events, so their data were excluded from the analysis. For each participant we calculated the average response to each measure across all eight events and use these as inputs in the regression analysis.

\section{Results and Discussion}

Participants estimated that events occurred on average three months prior to their actual occurrence $(M=2.8, t(180)=2.51, p<.05)$. Estimates were not affected by markers $(F(1,179)$ $=1.52, p>.2$ ), an effect that becomes even weaker when controlling for emotionality and memorability $(F(1,177)<1)$. We subsequently control for estimated months in order to establish the unique effect of markers on subjective feelings of elapsed time, over and above other dating biases. 
We regressed participants' subjective feelings of elapsed time on order of events in the survey, memorability, emotionality, and perceived number of months elapsed since the target event. Order of events $(F(1,175)=0.86, p>.3)$ and estimated months passed $(F(1,175)=1.20$, $p>$.25) did not exert a significant effect on participants' subjective feeling of time. In line with prior research, more memorable $(b=-0.4, F(1,175)=2.58, p<.12)$ and, particularly, more emotional events $(b=-0.6, F(1,175)=7.69, p<.01)$ were perceived as having occurred more recently. Most critically, controlling for memorability, emotionality and estimated dates, the extent to which target events were perceived as having triggered subsequent events exerted a strong effect on participants' subjective feelings of elapsed time $(F(1,175)=15.50, p<.001)$. Supporting our prediction, the more subsequent events (i.e., markers) that were perceived to have been triggered by the target events, the more distant the events felt $(b=0.4)$.

Our findings indicate that the perceived number of subsequent events triggered by a target event has a significant effect on how distant the target feels. The target events in this study were known, public events that varied in the number of subsequent events that they triggered. As such, this study offers an ecologically valid test of our hypothesis, and shows that the effect of markers is independent of other, previously-document dating biases.

\section{Study 2: College Admission Letter}

Our interpretation of the results of study 1 is predicated on the belief that participants accessed event markers when thinking about the elapsed time since a target event. In order to test the causal effect of accessing event markers more directly, in this study we manipulate the number of accessible markers associated with a highly significant life event: the day participants received their letter of admission to college. Participants were asked to recall either few or many 
events that were precipitated by their university admission and then to indicate their feeling of elapsed time since receiving the admission letter. They either wrote down these events or, in a separate follow-up study, were simply prompted to think about these events.

\section{Methods}

One hundred and three University of Southern California (USC) undergraduate students (75 female, 28 male) completed this study in return for candy. Participants were asked to think about the day they received their admission letter and then instructed to write down either two (low marker condition, $n=51$ ) or eight (high marker condition, $n=52$ ) events that were precipitated by the letter. All participants followed instructions and wrote down two or eight events, respectively. Next, they indicated their subjective feeling of elapsed time since receiving the admission letter on a 15-point scale anchored by “feels very recent” (1) and “feels very distant” (15), how well they remembered the day that they received the letter on a 7-point scale ranging from "not at all” (1) to "perfectly" (7), and how emotional the day was on a 7-point scale anchored by "not at all emotional” (1) and “extremely emotional” (7). Our manipulation did not affect emotionality or memorabilty. Finally, they were asked to report the approximate month and year when they received their admission letter.

\section{Results and Discussion}

We regressed feeling of elapsed time on marker condition, memory for the event, and emotionality. Emotionality was not a significant predictor of feelings of elapsed time $(F(1,99)<$ 1), but greater memorability was associated with a greater feeling of recency $(b=-0.6, F(1,99)=$ 4.44, $p<.05)$. More importantly, we find a significant effect of our marker manipulation, such 
that respondents who recalled eight event markers $(M=10.3)$ felt that the target event was more distant than those who recalled only two event markers $(M=8.9, F(1,99)=6.95, p<.01)$.

Among participants who reported a date for when they received their admission letter ( $N$ = 86), estimated months passed differed slightly between groups $\left(M_{2 \text { events }}=30.3, M_{8 \text { events }}=34.5\right.$, $F(1,84)=2.55, p=.11)$. However, when added to the regression model, reported months passed did not exert a significant effect on feelings of elapsed time $(F(1,81)=0.67, p>.4)$.

Furthermore, the effects of event memorability $(b=-0.89, F(1,81)=8.25, p<.01)$ and number of markers recalled $(b=1.45, F(1,81)=4.25, p<.05)$ remained significant, which supports our hypothesis.

An alternative interpretation of our data is that generating eight markers was more metacognitively difficult than generating two markers, and that this perception of greater retrieval difficulty is causally related to greater feelings of elapsed time (e.g., Schwarz 2005). In order to replicate our findings and address this alternative, in a follow-up study we cut the number of required markers in half and simply asked participants to think about these events without writing them down; both changes were designed, in part, to reduce the difficulty of the task. Although asking participants to merely think about the events sacrificed some experimental control, this thought task better approximates the intuitive, implicit process we propose that people engage in when assessing feelings of elapsed time. In this study we asked University of Pennsylvania undergraduates (62 male, 67 female) to think about one $(n=63)$ versus four ( $n=$ 66) events that were triggered by their admission letter to the university. ${ }^{1}$

\footnotetext{
${ }^{1}$ We also conducted a similar study asking USC undergraduates (63 male, 20 female) to simply think about two ( $\mathrm{n}=$ $41)$ versus eight $(n=42)$ events, the same number of events as the original study. Our results parallel both studies reported above in that prompting participants to think about eight events $\left(\mathrm{M}_{8}\right.$ events $\left.=9.8\right)$ made participants feel the target event had occurred longer ago $\left(M_{2 \text { events }}=8.7, F(1,76)=3.79, p=.05\right)$ over and above perceptions of difficulty $(F(1,76)<0.3)$. For those who reported a date for when they received their admission letter $(N=80)$, reported months passed did not have a significant effect on feelings of elapsed time when added to the model $(F(1,72)<1)$, but the number of events still affected feelings of elapsed time $(F(1,72)=3.64, p=.06)$.
} 
Our manipulation did not affect the perceived difficulty of generating markers $(F(1,127)$ $<1)$. Controlling for difficulty in our model, we find that neither emotionality $(F(1,124)<0.1)$ nor difficulty $(F(1,124)<0.4)$ were significant predictors of feelings of elapsed time, but memorability was $(b=-0.9, F(1,124)=14.44, p<.01)$. Most importantly, we replicate our findings: prompting participants to think about a greater number of events made them feel that the target event had occurred longer ago $\left(M_{1 \text { event }}=9.3, M_{4 \text { events }}=11.1, F(1,122)=8.28, p<.01\right)$.

As before, our manipulation directionally affected the reported date of receiving the admission letter $\left(N=99, M_{1 \text { event }}=25.9, M_{4 \text { events }}=29.4, F(1,100)=1.98, p=.16\right)$. However, controlling for estimated months passed $(b=0.06, F(1,93)=4.5, p<.05)$ did not alter the effect of the accessibility manipulation on feelings of elapsed time $(F(1,93)=5.33, p<.05)$.

Our results suggest that the number of accessible event markers affects subjective feelings of elapsed time. For the same event, respondents who were asked to recall a greater number of markers felt that receiving their admission letter was more distant, whereas those who were asked to recall fewer markers felt that the event was more recent. By manipulating the number of markers brought to mind, we demonstrate that feelings of elapsed time are influenced by the accessibility of event markers and that this effect is independent of memorabilty, emotionality, difficulty, and any effect of dating events.

\section{Study 3: Short Film}

In our first two studies we relied on participants' memories of events and markers that they had previously experienced. In this study we created an actual target event for participants to experience and experimentally manipulated the number of subsequent events triggered by this target event. The target event consisted of selecting and watching a short, humorous film during a laboratory session. The subsequent event markers consisted of follow-up emails that were sent 
over the course of a month and that included short tasks related to the film selected. We purposely chose a target event and event markers that were trivial compared to participants' other day-to-day experiences so that our task would represent only a tiny proportion of their activities during the month-long experiment. Thus, any difference between conditions in the number of follow-up tasks would exert a negligible effect on how "full" participants' subsequent weeks were (cf. Ornstein, 1969), enabling us to attribute any differences in feelings of elapsed time to the influence of event markers.

\section{Methods}

To create the initial target event, University of Pennsylvania undergraduates who participated in an hour long session, also completed an approximately 10 minute-long laboratory study where they watched one 3-4 minute comedic film, ostensibly to calibrate a film recommendation system. After viewing the film, they rated its humor, creativity, and technical quality and also indicated how much they had enjoyed the film, how emotional it was to them, and how memorable the film would be to them in the future. From this pool of undergraduates ( $n=131$ ), we recruited participants who would be willing to complete follow-up tasks sent via email. Our analyses are based on the 40 participants who completed all follow-up tasks and the final rating task. Although this constitutes a high mortality rate (70\%) relative to those who participated in the original lab study, it is not unexpected given the longitudinal nature of the experiment and the fact that participants were not informed about the nature and frequency of these follow-up tasks a priori. The latter was important in order to ensure the validity of the study, but resulted in participants entering the study without realizing the full demands of the longitudinal task. 
For the purpose of the follow-up task participants were randomly assigned to receive either one follow-up task or four follow-up tasks. Note that mortality was equivalent across experimental conditions resulting in approximately equal sample sizes ( $\left.n_{1 \text { event }}=18, n_{4 \text { events }}=22\right)$, and should not interact with our manipulation. Yet, due to our relatively small sample size and participant mortality we interpret our results with caution.

To simulate event markers that would appear to be triggered by the initial task, the follow-up tasks probed participants for various thoughts specifically related to the initial film preference task. For the first task (3 days after the film), participants described their ideas for further plots of the film. The second task (7 days after the film) asked participants to list other films in the same genre of comedy. In the third task (11 days after the film) participants provided characteristics of the target audience for their chosen genre of comedy. In task four (14 days after the film) participants explained how their chosen genre of comedy relates to their own personal experiences. Each task took approximately two minutes to complete. Participants in the one event marker condition completed only the fourth task on day 14 .

Twenty-seven days after the initial lab session, and two weeks after the last e-mail task, all participants were e-mailed the final survey that they had to complete within 48 hours. $^{2}$ They reported their subjective feeling of elapsed time since the initial film-viewing task, the film's memorability, and the film's emotionality. In addition, participants were asked to estimate the actual date, including month, day, and year, on which they participated in the initial film preference task in the lab. Note that the final survey made no reference to the intervening tasks.

\footnotetext{
2 The final survey was the second (one event marker condition) or, respectively, fifth (many event markers condition) email they received since the laboratory session.
} 


\section{Results and Discussion}

First, to examine participants' estimates of the date of the original task, we calculated estimated days passed as the difference between the judged date and the day the final task was administered. An accurate estimate would have been 27 days, yet participants' estimated dates were biased backward by about five days $(t(39)=2.67, p<.05)$. However, there was no difference in estimated days passed between conditions $(F(1,38)=0.63, p>.4)$.

Next, to test our marker hypothesis, we regressed subjective feeling of elapsed time on event markers condition, chosen film, estimated months past, and the emotionality and memorability reported in the final rating task. The analysis lent support to our hypothesis: more event markers marginally increased the subjective feelings of elapsed time $\left(M_{1}\right.$ event $=8.6, M_{4}$ events $=10.1, F(1,33)=3.07, p<.09)$. This was the case even after controlling for emotionality $(b$ $=-0.74, F(1,33)=4.46, p<.05)$, which as in experiments 1 and 2 was negatively associated with subjective feelings of elapsed time. ${ }^{3}$ Neither the chosen film $(F(2,33)=0.87, p>.4)$, memorability $(F(1,33)=1.14, p>.25)$, nor estimated months passed $(F(1,33)=2.22, p>.14)$ affected people’s subjective feelings.

Study 3 conceptually replicates studies 1 and 2 using a personal target event and experimentally controlled markers. Although our conclusions are clearly limited by the sample size and participant mortality, we believe that the findings of this study are noteworthy given the context of the experiment. Not only did students participate over an entire month without fully knowing what the follow-up tasks would entail, but also, given the host of other activities that they were involved in during that time, the target event and subsequent event markers were

\footnotetext{
${ }^{3}$ Note that we find a similar pattern for the effect of markers $(F(1,34)=3.91, p=.06)$ when controlling for the emotionality $(b=-0.67, F(1,34)=3.49, p=.07)$ and predicted memorability $(F(1,34)=0.01)$ ratings that had been reported in the initial lab session. The specific film did not have an effect on feelings of elapsed time $(F(1,34)=$ $0.92, p=.41)$.
} 
practically meaningless in their lives. It bears mentioning that the number of event markers influenced participants' subjective feeling of elapsed time, even when these markers were not made salient to them in the follow-up survey.

\section{General Discussion}

Time has been of great interest to scholars from a wide range of disciplines in the sciences and humanities. A common thread unifies the concept of time across all these disciplines: time is not an absolute concept; instead, it is subject to the perspective of the observer and is influenced by the events that take place in its course (e.g., Heidegger, 1992/1924). Similarly, peoples' intuitive perceptions of time also seem to be systematically influenced by an event's relative placement to and connection with other events in one's personal time continuum.

These systematic influences often lead to the perplexing sense that a very distant event feels much more recent, while a recent event feels much more distant. While prior research identifies certain characteristics of the event itself to explain this effect (e.g., emotionality and memorability), our investigation focuses on the characteristics of the time elapsed since the event. We propose that the feeling of distance from a target event depends on the number of events triggered by that target event. Our studies use a variety of approaches to demonstrate the effect of such event markers on feelings of elapsed time: we measure markers (study 1), manipulate the accessibility of markers (study 2), and create both the target event and its markers (study 3). The target events include public events as well as autobiographical events that took place between one month and two years prior to our studies. These events range from the more meaningful, such as receiving a college acceptance letter, to the more meaningless, such as 
watching a short humorous film. The fact that our predictions apply across such a wide range of settings demonstrates the importance of event markers in determining feelings of elapsed time.

Our marker hypothesis presents a novel mechanism to explain people's sense of elapsed time. In particular, our data cannot be explained by strength-of-memory models (Brown et al., 1985) or anticipated by research on the filled-duration illusion (Ornstein, 1969). Furthermore, in all our studies we measure and control for those characteristics of a target event that contribute to the dating biases established in previous research. The fact that we are able to replicate these past results, yet still find an independent effect of event markers, attests to the robustness of event markers as determinants of time perception. Moreover, we demonstrate that the effect of markers on subjective feelings of elapsed time holds even after controlling for the estimated date of the events. This is important because it further distinguishes our effect from other dating and duration biases, and suggests that feelings of elapsed time are psychologically distinct from previously studied judgments of duration or date estimation.

In addition to being a commonly used expression, feelings of elapsed time may be particularly important inputs for decisions about timing behaviors. For example, when deciding when to donate again, people may assess how long ago it feels that they donated to a certain cause. As charities often send follow-up materials to past donors, such mailings may play the role of event markers. Donors who received a greater number of mailings might feel as if their previous donation occurred in the distant past. Consequently, they might hasten to donate again compared with donors who received fewer mailings. We leave this and other possible applications of the effects of event markers on feelings of elapsed time to future research. 


\section{REFERENCES}

Avni-Babad, D. \& Ritov I. (2003). Routine and the perception of time. Journal of Experimental Psychology: General, 132 (4), 543-550.

Block, R. A. (1974). Memory and the experience of duration in retrospect. Memory and Cognition, 2, 153-160.

Block, R. A. \& Zakay, D (1997). Prospective and retrospective duration judgments: A metaanalytic review. Psychonomic Bulletin \& Review, 4 (June), 184-197.

Bratfisch, O., Ekman, G., Lundberg, U., \& Kruger, K. (1971). Subjective temporal distance and emotional involvement. Scandinavian Journal of Psychology, 12 (3), 147-60.

Brown, N. R., Rips, L. J., \& Shevell, S. K. (1985). The subjective dates of natural events in verylong-term memory. Cognitive Psychology, 17 (2), 139-77.

Burt, C. D. \& Kemp S. (1991). Retrospective duration estimation of public events. Memory \& Cognition, 9 (3), 252-262

Faro, D., Leclerc, F. \& Hastie R. (2005). Perceived causality as a cue to temporal distance. Psychological Science, 16(9), 673-677.

Friedman, W. J. (1993). Memory for the time of past events. Psychological Bulletin, 113(1), 4466.

Friedman, W. J. (1996). Distance and location processes in memory for the times of past events. In D. L. Medin (Ed.), The psychology of learning and motivation: Advances in research and theory (pp. 1-41, Vol. 35). San Diego, CA, US: Academic Press. 
Heidegger, M. (1992). The concept of time. (W. McNeill. Trans.). Oxford: Basil Blackwell. (Original work published in 1924).

Morwitz, V. G. (1997). It seems like only yesterday: The nature and consequences of telescoping errors in marketing research. Journal of Consumer Psychology, 6 (1), 1 - 29.

Ornstein, R. E. (1969). On the experience of time. Harmondsworth, U.K.: Penguin.

Schwarz, N. (2005). When thinking feels difficult: Meta-cognitive experiences in judgment and decision making. Medical Decision Making, 25, 105-112.

Zakay, D. \& Block R. A. (2004). Prospective and retrospective duration judgments: an executive-control perspective. Acta Neurobiologiae Experimentalis, 64, 319-328. 


\section{ACKNOWLEDGMENTS}

The authors thank Daniel Read for his discussion of some of the ideas presented in this paper and Jim Bettman for helpful comments on a previous draft of the paper. The authors contributed equally to the manuscript. 


\section{$\underline{\text { Appendix }}$}

Public Events Used in Study 1

Actual dates and number of months passed by the time the study was conducted in parentheses.

1. Supreme Court Chief Justice John Roberts is confirmed in the Senate (September 29, 2005; 16 months)

2. Pope John Paul II dies (April 2, 2005, 21 months)

3. Brad Pitt and Jennifer Aniston begin divorce proceedings (March 26, 2005; 22 months)

4. Lance Armstrong wins his final Tour de France (July 24, 2005; 18 months)

5. Terrorist bombings in London subway (July 7, 2005;18 months)

6. Tom Cruise professes his love for Katie Holmes on Oprah (May 26, 2005; 20 months)

7. Palestinian Authority Chairman Yasser Arafat dies (November 11, 2004; 26 months)

8. Condoleezza Rice is confirmed as US Secretary of State (January 26, 2005; 24 months)

Note - The actual dates of the events were not given to participants. 